\title{
The Lack of Health Education and Toxoplasmosis (Zoonotic) that Can Risk both the Fetus and Mother Health
}

\author{
Doc. LinditaTerolli \\ University“Fan.S.Noli”, Korce, Albania \\ E-mail. lindita.terolli@yahoo.com
}

\section{Doi:10.5901/ajis.2013.v2n9p34}

\section{Abstract}

\begin{abstract}
Toxoplasmosis is a disease that presents a really great risk. It is the word about the most diffuse zoonosis in the world. Toxoplasmosis "Gondi" produces oocysts in the intestines of cats. The oocysts are egg cells too resistant that are released in the outer environment and present the infecting agent. This infecting agent can be taken directly by people with unwashed hands. As well as the ingestion by other animals that can form cysts which stay for months and years in their muscles. Even if the meat of these animals becomes an infection source or it is consumed without being well thermally processed. Touching the meat without gloves by the pregnant women, especially in the first trimestral of pregnancy is another transmission method that brings consequences for the fetus, in the $25 \%$ of the cases. The aim of the study is the identification of the problems and health education. Material and method: The type of the study: descriptive, the direction of the study retrospective. There were analyzed the medical files of the patients in the gynecology ward of the maternity of Korca. There were analyzed the complications. Interviews were conducted for the health education level of the population. Conclusions and results: The poor health education.
\end{abstract}

Keywords: Toxoplasmosis, abortions, malformations, strabismus, blindness.

\section{Introduction}

Toxoplasma is an infection which can bring to toxoplasmosis, and can be transmitted by cats to humans, the greatest danger is for pregnant women. That among many malformations analyzed below can bring abortion. As well as cats it can touch mamals and birds too and is caused by a parasite called Toxoplasma gondii. The fully developed disease is rare while the toxoplasma infection is very common. Its importance consists in the fact that humans as well as other warm-blooded animals can get it. Toxoplasma infection can be transmitted to humans by domestic, wild, and feral cats. The disease toxoplasmosis is caused by the microscopic protozoa Toxoplasma gondii. This disease is spread all over the world. According to some estimates around $30 \%$ of the world population is infected. For example, in Germany and France most people carry the parasite, whereas in South Korea it is quite rare. While 60 million people in the United States are said to be infected. Toxoplasmosis is commonly without symptoms, because our immune system keeps the parasite from causing illness. To people with weakened immune and pregnant women it appears more problematic. The primary hosts appear to be cats while humans and warm-blooded animals the intermediate ones. Considering this Toxoplasma gondii is not a pure human parasite.

Toxoplasma gondii is famous to change the host's behavior. Studies show that this parasite can make rats fearless near cats. This indicates the latest need for Toxoplasma gondii to get inside felines. The parasite enter the primary host while a rat is eaten by a cat. There have been a few studies with humans, too. Some results talk about a strong connection between schizophrenia and toxoplasmosis. Studies have shown that as a result of this parasite humans react more slowly. Some other studies show that women that have the parasite have a greater probability to cheat their husbands. Whereas men become more aggressive.

Humans get infected by:

- blood transfusion or organ transplantation (rarely)

- ingesting water or anything else that has been contaminated with cat feces

- consuming undercooked and infected meat (especially lamb, pork and venison)

- mother-to-child transmission. A pregnant woman, who has just been infected with Toxoplasma gondii can pass the infection to the fetus (congenital infection). Even though she might not have the symptoms the unborn baby may came in life and suffer from that disease. 


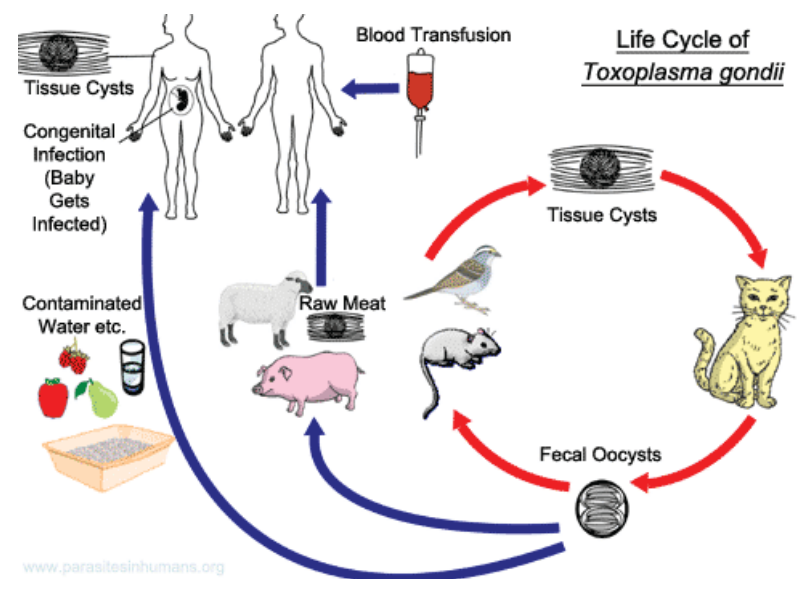

The life cycle of Toxoplasma gondii starts, when the resting form of the parasite-oocyst exit the primary host (cat) in the feces. Millions of oocysts are shed for as long as three weeks after infection. Only after a few days in the envirionment these become infective. These oocyst exist only in the feces of cats, both domestic and wild. Then when the water gets contaminated with these feces and is ingested by birds, humans or other intermediate hosts, they get the infection. (Healthy cats can get infected this way, too.) In the gut oocysts transform into tachyzoites which are about 4-8 $\mu \mathrm{m}$ long and 2-3 $\mu \mathrm{m}$ wide. They travel to other parts of the body via bloodstream and further develop into tissue cyst bradyzoites in muscle and neural tissue. The diameter of the cysts is about 5-50 $\mu \mathrm{m}$. They are commonly found in skeletal muscles, myocardium, brain and eyes where they can remain many decades. If a cat (or a human) eats the intermediate host, the tissue cysts get ingested and the parasite activates in the small intestine. People who are infected by this parasite may not have symptoms and this occurs because their immune system may keep these parasites from causing illness. 10-20 $\%$ of patients develop sore lymph nodes, muscle pains as well as other light symptoms that can last for weeks and then disappear (acute toxoplasmosis). The parasites remain in the body as tissue cysts (bradyzoites) and reactivate, if the person becomes immunosuppressed by other diseases or by immunosuppressive drugs. Usually if a woman has been infected before becoming pregnant, the unborn baby is safe because the mother has developed immunity. If a woman is pregnant and becomes infected with toxoplasmosis during or right before pregnancy, she can transmit the disease to her unborn child (congenital transmission). The effects are bigger if this transmission occurs earlier. And during all the period that the pregnancy goes on the infection continue to develop. This occurs as the result of the penetrability of the placenta. Symptoms might include:

$>$ miscarriage or stillborn baby

$>$ baby with brain or eye damage.

$>$ baby born with signs of toxoplasmosis such as abnormal enlargement or smallness of the head

$>$ It is the risk that even though at the very beginning the baby doesn't have any symptom, he can develop mental disability, vision loss and seizures later in life.

Congenital toxoplasmosis or infection after birth or rarely from acute toxoplasmosis as an adult can cause eye disease. According to the congenital infection eye lesions do not appear at birth but when the individual reach the adulthood, and this happens in $20-80 \%$ of the cases. In the restrict of Korca, Albania this occurs less than in $1 \%$ of the cases. The acute inflammatory lesion of the retina leaded by eye infection leaves retinochoroidal scarring. The symptoms of acute ocular toxoplasmosis:

Eye pain

Sensitivity to light-Photophobia

$>$ Tearing of the eyes

Redness of the eye

$>$ Reduced vision

But the reactivation of the eye disease later in life can cause even more damage to the retina. Especially if there are damaged the central structures of the retina that cause a progressive vision loss. Whereas people with weakened 
immune system may be more exposed to brain lesions, pneumonitis or retinochoroiditis, central nervous system disease among other risks. For example if we consider people with AIDS and renewed toxoplasmosis they may have symptoms such as:
$>$ Fever
$>$ Nausea
$>$ Confusion
$>$ Bad coordination
$>$ Headaches
$>$ Seizures

If a chronic toxoplasmosis reactivates in a pregnant woman that has a weakened immune who is infected before the pregnancy it might cause the congenital infection to the baby. The typical Toxoplasmosis diagnose is done by serologic tests by detecting immunoglobulin antibodies within several weeks of infection. Your health care provider examines your blood sample to find Toxoplasma-specific lgA, lgG or lgM antibodies. Living parasites can be also found in the sample (blood, cerebrospinal or other body fluids) but the process here becomes more complicated and difficult so this is not used. Direct observation of the parasite is possible in cerebrospinal fluid (CSF), stained tissue sections or other biopsy samples but these techniques are used less frequently due to their difficulty. A test that measures IgG determines if a person has been infected. If the person in examination is pregnant then here it is necessary to even determine the time of the infection. For this lgM is detected along with IgG avidity test. To detect Toxoplasma gondii DNA are use molecular techniques in the amniotic fluid in cases of congenital transmission . Ocular toxoplasmosis diagnosis is usually based on symptoms, appearance of lesions in the eye, serologic testing and course of the infection. Serologic tests can be unreliable in immunosuppressed patients. While as treatment to Toxoplasmisis can serve combinations of pyrimethamine with either trisulfapyrimidines or sulfadiazine, plus folinic acid in the form of leucovorin calcium to protect the bone marrow from the toxic effects of pyrimethamine. If this treatment causes hypersensitivity reaction, then pyrimethamine and clindamycin can be used instead. In case of unavailability of these drugs then a combination of sulfamethoxazole and trimethoprim can be used. Even though the pregnant women and babies can be treated the Toxoplasmosis gondii do not disappear completely. The parasites can remain within tissue cells in a less active stage (cyst) in locations difficult for the medication to get to. A drug called spiramycin is recommended during the first four months whereas sulfadizaine/pyrimethamine and folinic acid for women that have been pregnant for more than four months. PCR (a method to discover parasite DNA) is often performed on the amniotic fluid to find out if the infant is infected. If the infant is likely to be infected, then treatment is done with drugs such as sulfadizaine, pyrimethamine and folinic acid. Congenitally infected babies are treated with sulfonamide and pyrimethamine. While the treatment for persons with ocular disease depends on the size of the eye lesion, the characteristics (acute or chronic) and the location of the lesion. Persons with compromised immune systems (such as AIDS patients) need to be treated until their health improves significantly.

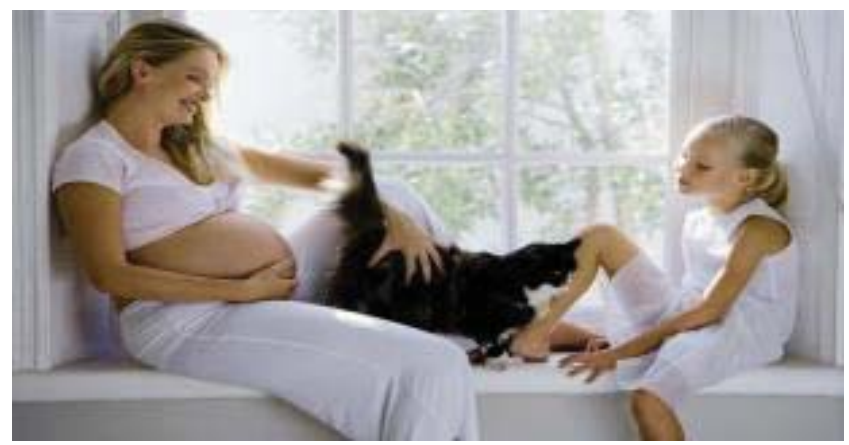

\section{Methods and results}

The survey is done by the medical personnel of Obsetric-Gynecology, Lab and Oculists. During the period 2009-2011. Through out questionnaires with suspected people was taken demographic and clinic data. Data about the serologic confirmation is taken in the laboratory of Korca restrict. The suspected and serologically confirmed newborns for Congenital Toxoplasmosis were treated clinically about one year. In the population of 342 pregnant women there were 
examined the serum samples and tested for $\lg G$, IgM and among them 170 women resulted positive about $\lg G$, while 5 of them (1.4\%) were positive for IGM. Six cases were presented with severe symptoms at birth. Choriretinitis was the main manifestation. The incidence was $0.1-1$ for 1000 births (alive ones). The prevalence of the birth was $49 \%$ in $2009,49.5 \%$ in 2010 and $49 \%$ in 2011. Or 5 in 10000 births.

A probable case of CT was defined as a child $<12$ months of age with unexplained hepatosplenomegaly or lymphadenopathy or any symptom of the classical triad (hydrocephalus, intracranial calcifications and chorioretinitis) and the presence of Toxoplasma IgG antibodies, with maternal serological results compatible with confirmed infection during pregnancy) or any asymptomatic infant $<12$ months of age with high levels of Toxoplasma lgG (>300 IU /ml) and maternal serology compatible with Toxoplasma infection during pregnancy.

\section{Recommendations}

- Restrict your pet cats from being in touch with rodents and birds, and give them only cooked meat, commercially prepared cat food, and pasteurized dairy products. Don't allow them to get food in garbage cans.

- Transmission of oocysts present in litter boxes or garden soil and anywhere cats may defecate can be avoided by wearing rubber gloves during contact with contaminated soil or litter.

- Wash your hands thoroughly after contact. Cover sandboxes to prevent cats from defecating in them. Get rid of feces from litter boxes every day, and disinfect them with hot water or dry heat; chemical disinfection is not effective in destroying oocysts

- During a present pregnancy or a planned one soon, casts must be tested for antibodies to Toxoplasma. Don't handle litter boxes. Stay away from free-roaming cats as they have the largest risk to be contaminated with oocysts. Be careful in way that any outdoor car doesn't get in contact with bedclothes and any furnishings in use. Don't handle a cat that seems ill. Wash uncooked vegetables thoroughly before eating. Wash hands thoroughly with soap and water after contact with soil and cats.

- To avoid these and other risks it is recommended health education by preconception advice.

\section{References}

(2009), Department of Public Health in Korca.

Engl, N . Med, J. (1992), Toxoplasmosis of the central nervous system in the acquired immunodeficiency syndrome.

Ho-Yen.D.O Human Toxoplasmis , (1992) , Oxford University Press

Kim.K \& Wiess.L.M , (2011) , Toxoplasma gondii, Academis Press Klein. JD (eds) 1983 Infectious Diseases of the Fetus and Newborn, p. 143-263, WB Saunders Co, Philadelphia

$(2009,2011)$,Maternity in Korce .

Perkins.E.S , Little Brown , 1961,Toxoplasmosis.

Remington JS, McLeod R, Thulliez P, Desmonts G. Toxoplasmosis. In: Remington JS, Klein JO, (2001). Infectious Diseases of the Fetus and Newborn Infant. 5th ed. Philadelphia, p. 205-346.

Thomas.P.A \& Petersen.E , 2000, Springer, Congenital Toxoplasmis. 